\title{
Warum Begriffe aus der Ökonomie uns Ärztinnen und Ärzte nicht erschrecken müssen!
}

\author{
med-swiss.net, die Dachorganisation der Schweizer Ärztenetzwerke mit
} 36 angeschlossenen Netzwerken und rund 1500 Ärztinnen und Ärzten*, möchte die neu angelaufene Diskussion über Managed Care (MC) in der Schweiz versachlichen. Der Beitrag soll Informationsmängel und Missverständnisse korrigieren und daraus entstehende Berührungsängste mit Care Management lösen.

\section{Philip Dreiding ${ }^{a}$, Jörg Fritschi ${ }^{b}$}

a Dr. med., Allgemeinarzt FMH, Zürich, Vorstand med-swiss.net

b Dr. med., Allgemeinarzt FMH, Obernau, Vorstand med-swiss.net

* Bei männlichen oder weiblichen Formen ist selbstredend immer auch das andere Geschlecht gemeint.
Korrespondenz:

Dr. med. Jörg Fritschi

Kleinholz 3

CH-6012 Obernau

\section{Was ist eine Ärztenetzwerk?}

Ärztenetzwerke sind Organisationen, die von Leistungserbringern gebildet werden und der Gesundheitsversorgung dienen. Durch verbindliches Zusammenwirken unter sich, mit netzfremden Leistungserbringern und mit den Kostenträgern erbringen sie auf die Bedürfnisse der Patientinnen und Patienten ausgerichtete Gesundheitsleistungen.

Das Zusammenwirken beruht auf vereinbarten Behandlungsprozessen, unternehmerischen Organisationsstrukturen und einer gemeinsamen Betreuungskultur. Ärztenetzwerke setzen sich bei hoher Versorgungsqualität einen haushälterischen Umgang mit den vorhandenen Mitteln zum Ziel.

Ärztenetzwerke sind bereit, ökonomische Verantwortung zu übernehmen und die Gesundheitsleistungen aus einer Hand zu steuern.

\section{Was kann ein Ärztenetzwerk bieten?}

- Die Patientenbetreuung erfolgt qualitäts- und nachweisbar kostenbewusst.

- Die Verantwortung wird durch strukturierte Kommunikationsformen (Qualitätszirkel, CIRS) breiter abgestützt. Die Behandlungssicherheit steigt.

- Durch verbindliche Abmachungen unter den einzelnen Mitgliedern und durch strukturierte innere Abläufe kann ein Ärztenetzwerk als stärkerer Partner gegenüber Versicherungen auftreten und die eigenen Interessen und diejenigen der Patienten gegenüber der Öffentlichkeit besser vertreten als die einzelne Ärztin oder der einzelne Arzt.
- Das Rationalisierungspotential bezüglich Informationsaustausch, Fortbildung und gemeinsamem Einkauf kann ausgeschöpft werden.

- Von den WZW-Kriterien (Wirksamkeit, Zweckmässigkeit, Wirtschaftlichkeit) kann vor allem letzteres von einem Ärztenetzwerk weit besser umgesetzt werden als von einzelnen Ärztinnen und Ärzten.

\section{Medizinische Leistungen und Ökonomie}

Angesichts der Kostenentwicklung im Gesundheitswesen und der politischen Grosswetterlage kann es sich die Ärzteschaft mittelfristig nicht länger leisten, die ökonomischen Aspekte ihrer Kerntätigkeit von Politikern und Ökonomen allein beurteilen und verwalten zu lassen.

Wer anders könnte die Wirksamkeit, die Zweckmässigkeit und selbstverständlich auch die Wirtschaftlichkeit der medizinischen Leistungen besser beurteilen als die Ärzteschaft selbst?

Ein Weg dazu ist die Teilnahme am Care Management, das mit oder ohne Ärzteschaft seinen Gang nehmen wird. Care Management setzt jedoch Verantwortungsbereitschaft voraus, auch auf ökonomischer Ebene.

Zentrale Elemente ökonomischer Verantwortung sind Begriffe wie Kollektiv, Budget und Risikobeteiligung.

\section{Das Kollektiv}

So banal wie es tönt, so wichtig ist es: Managed Care kann nur über die Kollektive der Versicherten und der Ärzte umgesetzt werden! Die Ärzteschaft muss sich dafür in einer geeigneten Gesellschaftsform organisieren (Verein, Aktiengesell- 
schaft), damit die einzelnen Mitglieder nicht mit ihrem Privatvermögen haften.

\section{Das Budget}

Ein Budget stellt eine Kostenzielvorgabe für die Betreuung eines Versichertenkollektivs dar und kann mit verschiedenen Methoden berechnet werden. Das Budget kann die ganze Behandlungskette oder nur einen Teil davon umfassen.

Wichtigstes Element der Behandlungskette ist die obligatorische Krankenpflege (OKP): ambulante Leistungen, stationäre Leistungen, Medikamente, Labor usw. Verträge über die ganze OKP sind vorzuziehen und haben sich eingebürgert, damit ambulant mögliche Behandlungen nicht stationär durchgeführt werden, wo sie schlussendlich höhere Kosten generieren. (Unter dem Gesichtswinkel des Verursacherprinzips wird die Behandlungskette in verursachte und veranlasste Leistungen unterteilt.)

Ein Budget kann grundsätzlich mit oder ohne Risikobeteiligung vereinbart werden. Ohne Risikobeteiligung und damit automatisch ohne ökonomische Mitverantwortung erlaubt es immerhin einen Kostenvergleich.

Merke: Nirgends in der Schweiz wird ein Budgetbetrag einer einzelnen Ärztin/einem einzelnen Arzt ausbezahlt!

\section{Gebräuchliche Budgetberechnungs- methoden}

\section{a) Vergleich des MC-Versichertenkollektivs mit einem konventionell versicherten Kollektiv (Parallelkostenrechnung)}

MC-Kollektive arbeiten günstiger! Lange war die Frage offen, ob die Kostendifferenz durch eine echte Einsparung oder durch Versichertenselektion zustande kommt. Die Haus-Arzt-Modellstudie Aarau (HAMA-Studie) [1] bewies, dass zwei in Zusammenarbeit mit den teilnehmenden Versicherern gebildete Modellkollektive bezüglich Alter und Morbidität vergleichbar waren. Die Einsparungen sind also echt!

\section{b) Vergleich des MC-Kollektivs mit seinen Vorjahreskosten (Vorkostenrechnung)}

Bei dieser Berechnungsmethode müssen vor allem das Durchschnittsalter des Kollektivs und die Teuerung unbedingt mitberücksichtigt werden.

c) Budget pro Versicherten und Jahr (Capitation, Kopfpauschale)

Die Basis dieser verfeinerten und deshalb auch aufwendigen Berechnungsmethode ist der Betrag,

\section{«Take home messages» für eilige} Leserinnen und Leser

1. Warum überlassen wir Ärztinnen und Ärzte das WZW-Kriterium Wirtschaftlichkeit allein den Ökonomen? Wer kann die Wirtschaftlichkeit unseres Handelns besser beurteilen als wir selber?

2. Managed Care wird immer nur über Kollektive (Versicherte, Patienten, Ärzte) umgesetzt.

3. Ein Budget ist ein verhandelbares Kostenziel.

4. Capitation (Kopfpauschale) ist lediglich eine von mehreren Budgetberechnungsmethoden. Capitation stellt keine Versicherungsform dar!

5. Ökonomische Mitverantwortung entsteht erst durch Risikobeteiligung. Risikobeteiligung besteht nicht nur aus Verlust, sondern auch aus Gewinn!

6. Risikobeteiligung ist verhandelbar und nicht in Stein gemeisselt. Sie kann prozentualisiert und limitiert werden. Am Risiko beteiligen sich Versicherer und Ärztenetzwerk! 7. Irrtümer und Fallgruben:

- Streichen Sie das Schlagwort «Budgetverantwortung» aus Ihrem Vokabular und ersetzen Sie es mindestens durch den die Realität besser abbildenden Ausdruck «Budgetmitverantwortung». Noch besser: Lesen Sie den ganzen Artikel! Im Detail liegt das Verstehen der Zusammenhänge.

- Vergessen Sie das Märchen, dass der einzelne Arzt ein Kopfgeld für die einzelne Patientin erhält! Vergessen Sie das Märchen, dass der budgetierte Betrag vor dem Jahresende «aufgebraucht» ist! Es gibt in der Schweiz unseres Wissens kein Ärztenetzwerk, das eine vollumfängliche ökonomische Verantwortung übernommen hätte, sich die Versicherungsprämie direkt auszahlen liesse und damit in die Rolle des Versicherers träte.

den ein einzelner Versicherter voraussichtlich im kommenden Jahr benötigt. Die Summe der Kopfpauschalen ergibt das Budget für das Kollektiv.

Die Kopfpauschale errechnet sich mit den folgenden Kriterien oder einer Kombination davon (Alter, Geschlecht, Vorjahreshospitalisation, Morbidität, Stadt-/Landgebiet, Medikamente, Teuerung usw.) für jeden Versicherten.

Die Budgetberechnung innerhalb des gleichen Kollektivs von Jahr zu Jahr schliesst den Selektionszufall gänzlich aus. 
Unter dem Begriff «Volle Capitation» wird die Berechnung einer Kopfpauschale über das ganze Leistungspaket der OKP (ambulant, stationär, verursacht, veranlasst) verstanden.

Merke: «Capitation» stellt keine Versicherungsform, sondern lediglich ein Instrument zur Budgetberechnung dar!

\section{Die Risikobeteiligung}

Entscheidet sich ein Ärztenetzwerk für eine ökonomische Mitverantwortung, muss es sich am unternehmerischen Risiko beteiligen. Risiko heisst: Gewinn oder Verlust (aber nicht nur Verlust!).

Die Risikobeteiligung ist das zentrale Element ökonomischer Mitverantwortung. Dabei gilt eine einfache Regel der Ökonomie: Je höher das gewählte Risiko, desto grösser sind möglicher Gewinn und möglicher Verlust.

Die Risikobeteiligung wird vertraglich zwischen Ärztenetzwerk und Versicherer geregelt. Sie wird zwischen beiden Partnern prozentual aufgeteilt und kann auch betraglich limitiert werden.

Weitere Sicherungsmassnahmen können vorgesehen und vertraglich geregelt werden (Rückstellungen, Rückversicherungen, Grossrisikoabfederung).

Bleibt der budgetierte Betrag beim Versicherer und wird dem Ärztenetzwerk nicht überwiesen, so spricht man von virtuellem Budget. Die ökonomische Mitverantwortung des Ärztenetzwerkes beschränkt sich damit in erster Linie auf die Einhaltung der Kostenvorgabe.

Wird der budgetierte Betrag dem Ärztenetzwerk physisch ausbezahlt (ausbezahltes Budget), wird das Ärztenetzwerk auch für die Leistungskontrolle, den Zahlungsverkehr und die dafür notwendigen Reserven verantwortlich. Der Versicherer ist noch für die Prämien und für das Grossrisikomanagement zuständig. Nach wie vor beteiligt er sich jedoch vertraglich geregelt am Risiko.

(Eine vollständige ökonomische Verantwortung des Ärztenetzwerkes würde dann vorliegen, wenn das Risiko vollumfänglich vom Ärztenetzwerk getragen würde. Das Netzwerk tritt in die Rolle des Versicherers! Ein solches Netzwerk existiert unseres Wissens in der Schweiz nicht.)

Nach dem Gesagten versteht es sich von selbst: Eine professionelle Beratung und Führung des Ärztenetzwerkes durch einen fachlich kompetenten Ökonomen, der sich im Gesundheitswesen und in der Versicherungsbranche gleichermassen auskennt und der in die ökonomische Mitverantwortung ebenso einbezogen ist wie das Ärztenetzwerk, ist unumgänglich.

\section{Welche Managed-Care-Vertragsformen} sind heute in der Schweiz gebräuchlich?

\section{a) «Einfacher» MC-Vertrag ohne ökonomische Verantwortung}

Zwischen Versicherer und einem Ärztenetzwerk wird ein Zusammenarbeitsvertrag mit den drei folgenden Elementen ausgehandelt:

\section{Gatekeeping}

Der Patient meldet sich immer zuerst bei seinem aus dem Ärztenetzwerk ausgewählten Hausarzt.

\section{Information des Ärztenetzwerkes über die Kostenentwicklung}

Der Versicherer stellt dem Ärztenetzwerk Kenndaten der Kostenentwicklung zur Verfügung. Die Kenndaten der einzelnen Netzwerkmitglieder sind transparent.

\section{Überweisungskontrolle}

Das Gatekeeping wird vom Netzwerk kontrolliert.

Der Steuerungsaufwand des Netzwerks und allfällige freiwillige Projektarbeit werden von den Versicherern anteilmässig vergütet.

Üblicherweise werden die Kosten des MCKollektivs mit den Kosten eines konventionell versicherten Kollektivs (Parallelkosten) oder mit seinen eigenen Vorjahreskosten (Vorkosten) verglichen (vgl. oben «Das Kollektiv»).

Aus diversen Gründen hat sich eingebürgert, dass das daraus errechnete Budget selten mit einer Risikobeteiligung «scharfgestellt» wird (was ja einer ökonomischen Mitverantwortung entsprechen würde).

Immerhin generiert ein MC-System auch ohne ökonomische Verantwortung gemäss der HAMA-Studie bereits eine Kostenreduktion von rund $12-15 \%$ bei vergleichbarem Versichertenkollektiv. Ob es sich bei den versicherten Patienten und Patientinnen um besonders sparsame Personen handelt, ist letztlich egal. Warum sollten wir den einsichtigen und kooperativen Patienten und die Patientinnen nicht zu günstigeren Prämien verhelfen?

\section{Cavete}

Neuerdings bieten gewisse Krankenversicherer unter dem Namen «Hausarztversicherung» als Marketingmittel flächendeckende Listenversicherungsprodukte an, ohne dass ein verbindlicher Vertrag mit einem Ärzteverein ausgehandelt wurde. Diese Modelle haben mit Managed Care nichts zu tun! 
b) MC-Verträge mit teilweiser ökonomischer Verantwortung

Der Vertrag umfasst dieselben Elemente wie der einfache MC-Vertrag (Gatekeeping, Kostentransparenz, Überweisungskontrolle); das mit einer der obgenannten Methoden errechnete Budget wird jedoch im Rahmen einer Risikobeteiligung scharfgestellt.

In der Schweiz hat die Budgetberechnung auf der Basis der Capitation Verbreitung gefunden. Die Berechnungsmethode mit Hilfe der Parallelkosten oder der Vorkosten steht bei MC-Verträgen mit ökonomischer Mitverantwortung im Hintergrund.

Das Ärztenetzwerk und der Versicherer beteiligen sich vertraglich geregelt am Risiko (Gewinn/Verlust). Die Budgetverantwortung ist zwischen Versicherer und Ärztenetzwerk gleichsam aufgeteilt. Deswegen drängt sich die Bezeichnung «Budgetmitverantwortung» auf.

Der umgangssprachlich salopp verwendete Begriff «Budgetverantwortung» bildet die Realität schlecht $a b$, hat bei der Ärzteschaft zu manchen Missverständnissen geführt und dem Ruf des Care Managements sehr geschadet hat.

Wieso sind MC-Verträge mit Budgetmitverantwortung eine wirksame Methode, die Ärzteschaft vom wiederholt erhobenen Vorwurf der Mengenausweitung und der Überarztung zu befreien?

Die Ärzteschaft verrechnet ihre Leistungen den Patienten oder dem Versicherer wie üblich nach dem Einzelleistungsprinzip im Rahmen der gültigen Tarifvereinbarung.

Die Einzelleistungsverrechnung beinhaltet die latente Tendenz der Überversorgung bzw. der von den Versicherern immer wieder heraufbeschworenen Mengenausweitung. (Selbst wenn tatsächlich keine Mengenausweitung stattfindet, so kann dieser Vorwurf ärztlicherseits nicht mit strikten Beweisen widerlegt werden.)

Umgekehrt wohnt einem alleinigen Budget die latente Tendenz der Unterversorgung bei.

Budgetmitverantwortungsverträge kombinieren die Einzelleistungsverrechnung mit der Budgetkontrolle und gleichen damit beide Tendenzen elegant aus:

Belastet ein Netzwerkmitglied mit den verrechneten Einzelleistungen das Netzbudget über Gebühr, schuldet es den anderen Mitgliedern im Rahmen gegenseitiger Transparenz Rechenschaft. Andererseits wird wohl kaum ein Netzwerkmitglied an einem durch Unterversorgung entstehenden Einkommensverlust interessiert sein.

Auch das Netzwerk selber wird eine Unterversorgung tunlichst vermeiden; nur durch ange- messene Betreuung lassen sich kostenintensive Komplikationen bannen.

\section{MC-Verträge mit virtuellem Budget \\ («virtuelle Capitation»)}

Mit diesem Vertrag arbeiten gegenwärtig fünf Ärztenetzwerke in der Deutschschweiz.

Da der budgetierte Betrag nicht vom Netzwerk verwaltet wird, besteht kein «Risiko des aufgebrauchten Budgets». Für die Leistungskontrolle und die Reservebildung ist weiterhin der Versicherer verantwortlich.

Wird das Budget überschritten, wird der vertraglich definierte Fehlbetrag dem Netzwerk belastet. Weder die Patientinnen/Patienten noch die einzelnen Mitglieder des Ärztenetzwerkes tragen die Folgen persönlich.

\section{MC-Vertrag mit ausbezahltem Budget} («ausbezahlte Capitation»)

Mit diesem Vertrag arbeitet gegenwärtig ein Ärztenetzwerk in der Deutschschweiz.

Der Vertrag erfüllt dieselben Kriterien wie der MC-Vertrag mit virtuellem Budget. Der budgetierte Betrag wird jedoch physisch ausbezahlt, wodurch das Ärztenetzwerk für den Zahlungsverkehr der vertraglich definierten Leistungen der Behandlungskette und für die entsprechenden Rückstellungen verantwortlich wird. Das Honorar der einzelnen Ärztin und weiterer Leistungserbringer (Spezialisten, Spital, Physiotherapie, Spitex usw.) wird vom Netzwerk ausbezahlt.

Der Versicherer kontrolliert den Prämieneingang, übernimmt die zwingend notwendige Grossrisikoabdeckung, beteiligt sich aber nach wie vor vertraglich geregelt am Risiko.

Im Verhältnis zum unternehmerischen Risiko sind die unternehmerischen Freiheiten entsprechend hoch.

Werden die Mitglieder durch das Ärztenetzwerk mit einem pauschalen Lohn (wie bei einer versicherungseigenen $\mathrm{HMO}$ ) und nicht gemäss dem Einzelleistungsprinzip honoriert, besteht die Gefahr der Unterversorgung, die durch eine befohlene Qualitätssicherung (top down) aufgefangen werden muss.

\section{Häufig gestellte Fragen}

\section{Ist Qualität und Budgetmitverantwortung vereinbar?}

Durch optimale ambulante Versorgungsqualität lassen sich Hospitalisationen und damit hohe Folgekosten vermeiden. Eine Hospitalisation zur richtigen Zeit verhindert Komplikationen und damit weitere Kosten. Die optimale Zusammenarbeit zwischen Grundversorgern und Speziali- 
sten erhält im Rahmen der ökonomischen Mitverantwortung ganz besonderes Gewicht.

Damit relativiert die Budgetmitverantwortung mindestens einen Teil der von verschiedenen Obrigkeiten geforderten Obligatorien der Qualitätssicherung (Top-down-Qualität). Das Ärztenetzwerk wird im Rahmen eines selbstgewählten Qualitätsmanagements (Bottom-up-Qualität) von sich aus eine optimale ambulante Versorgung anstreben, um weit höhere Folgekosten zu vermeiden.

\section{Was passiert, wenn das Budget überschritten wird?}

Wird im Rahmen des virtuellen Budgets das Kostenziel überschritten, muss kontrolliert werden, ob dessen Berechnung korrekt erfolgt ist. Ist dies der Fall, so wird der vertraglich vereinbarte Anteil des Fehlbetrages ins anschliessende Geschäftsjahr des Netzwerkes übertragen.

Ist im Rahmen des ausbezahlten Budgets der Betrag aufgebraucht, muss das Ärztenetzwerk auf eigene Reserven zurückgreifen können.

Zwingen die Versicherer den Ärztenetzwerken ein ruinöses Budget auf, werden diese insolvent. Dies hätte zur Folge, dass sich die Zusammenarbeit zwischen Versicherern und Netzwerken auflöst. An einer solchen Entwicklung sind die Versicherer nicht interessiert, da sie damit Partner verlieren, die sich den ökonomischen Herausforderungen im Gesundheitswesen stellen

\section{Wie wird ein Budgetüberschuss im Rahmen der Budgetmitverantwortung gehandhabt?} Der Vertrag muss regeln, dass Überschüsse grundsätzlich im Versicherungssystem verbleiben und wie diese zwischen Versicherten, Versicherer und Ärztenetzwerk zu verteilen sind. Die Auflagen im KVG sind zu berücksichtigen.

Ärztenetzwerke mit ökonomischer Mitverantwortung weisen erfahrungsgemäss regelmässig einen Gewinn aus, der zur Prämienreduktion beiträgt und eine Entschädigung für die Netzwerkarbeit (z. B. Qualitätszirkelarbeit) und die Netzwerkentwicklung (z. B. Praxiszertifizierung) erlaubt.

\section{Fazit}

Die Budgetmitverantwortung ist ein erfolgversprechendes Zusammenarbeitsprinzip zwischen Ärztenetzwerken und Versicherern. Es ist eine zukunftsweisende Chance für die Ärzteschaft, das Gesundheitssystem mitzugestalten.

Nutzen wir diese Chance!

Für die Durchsicht mit entsprechenden Anregungen und Ergänzungen danken die Autoren den folgenden Personen ganz herzlich:

- Dr. med. Ueli Bodmer, Allgemeinarzt FMH, Geschäftsführer FIHAM (Fakultäre Instanz für Hausarztmedizin), Vorstandsmitglied medswiss.net;

- Dr. med. Max-Albrecht Fischer, Allgemeinarzt FMH, Präsident med-swiss.net;

- Karl Metzger, MC-Leiter BlueCare AG;

- Dr. med. Alexander von Weymarn, Radiologie FMH, leitender Arzt Spital Frauenfeld, Vizepräsident med-swiss.net;

- Christopher Züllig, Ökonom MediX Management;

- Dr. med. Hans Zaugg, Handchirurgie FMH, Hospitant Vorstand med-swiss.net.

\section{Literatur}

1 Hausarztmodellstudie Aarau (HAMA). http://jech. bmjjournals.com/cgi/content/full/60/1/ oder www.med-swiss.net. Die Studie wurde vom Verein Hausärzte der Region Aarau, der Abteilung medizinische Ökonomie des Universitätsspitals Zürich (Leitung: Prof. Thomas D. Szucs), der SanaCare AG und den Krankenversicherern Atupri, Concordia, Helsana und Wincare durchgeführt. 